\title{
1,3,4-噻二唑三氮烯化合物的合成和生物活性研究
}

\author{
张明千刘斌凯雷强颜景东赵中玉 龙 跃* \\ (郑州大学化学与分子工程学院 郑州 450001)
}

\begin{abstract}
摘要 利用拼接原理将药效基团三氮烯与 1,3,4-噻二唑相拼接，合成了 15 个未见报道的 1,3,4-噻二唑三氮烯类衍生物, 并用核磁共振波谱(NMR)、红外光谱(IR)和高分辨质谱(HRMS)等方法确定化合物结构. 通过以典型三氮烯药物达卡巴 嗪(DTIC)和药物 5-氟尿嘧啶(5-FU)作参照, 对人食管癌细胞(EC109)、人胃癌细胞(MGC803)和人前列腺癌细胞(PC-3) 做活性检测, 结果显示部分化合物对人胃癌细胞(MGC803)的抑制作用强于达卡巴嗪(DTIC), 其中 2-(3-甲基苯胺 基)-5-[4-(3,3-二甲基三氮烯-1-基)苯基]-1,3,4-噻二唑(8c)，2-(2-甲氧基苯胺基)-5-[4-(3,3-二甲基三氮烯-1-基)苯基]-1,3,4噻二唑(8f)，2-(3,4-二氯苯胺基)-5-[4-(3,3-二甲基三氮烯-1-基)苯基]-1,3,4-噻二唑(81)的 $\mathrm{IC}_{50}$ 值低于 5-氟尿嘧啶, 分别为 5.3, 6.5 和 $6.3 \mu \mathrm{mol} / \mathrm{L}$; 部分化合物对人前列腺癌细胞(PC-3)的抑制作用强于达卡巴嗪(DTIC), 其中 81 的 $\mathrm{IC}_{50}$ 值低于 $5-$ 氟尿嘧啶, 为 $13.5 \mu \mathrm{mol} / \mathrm{L}$.
\end{abstract}

关键词 三氮烯; 1,3,4-噻二唑; 人食管癌细胞; 人胃癌细胞; 人前列腺癌细胞

\section{Synthesis and Biological Activities of 1,3,4-Thiadiazole Triazene Compound}

\author{
Zhang, Mingqian Liu, Binkai Lei, Qiang Yan, Jingdong \\ Zhao, Zhongyu Long, Yue* \\ (College of Chemistry and Molecular Engineering, Zhengzhou University, Zhengzhou 450001)
}

\begin{abstract}
Using the splicing principle of combining the pharmacological group triazene with 1,3,4-thiadiazole, 15 unreported 1,3,4-thiadiazole triazene derivatives were synthesized. The structures of the compounds were determined by nucleated magnetic resonance spectroscopy (NMR), infrared spectroscopy (IR) and high-resolution mass spectrometry (HRMS). By using the typical triazene drug dacarbazine (DTIC) and drug 5-fluorouracil (5-FU) as a reference, the activity detections of human esop-hageal cancer cells (EC109), human gastric cancer cells (MGC803), and human prostate cancer cells (PC-3) were carried out. The results showed that some compounds inhibited human gastric cancer cells (MGC803) more strongly than dacarbazine (DTIC), and the $\mathrm{IC}_{50}$ values of compounds 2-(3-methyanilino)-5-[4-(3,3-dimethyltriazol-1-yl) phenyl]-1,3,4-thiadiazole (8c), 2-(2-methoxyanilino)-5-[4-(3,3-dimethyltriazol-1-yl) phenyl]-1,3,4-thiadiazole (8f), and 2-(3,4-dichloroanilino)-5-[4-(3,3-dimethyltriazol-1-yl) phenyl]-1,3,4-thiadiazole (8I) were lower than those of 5-fluorouracil with 5.3, 6.5 and $6.3 \mu \mathrm{mol} / \mathrm{L}$, respectively. Some compounds inhibited human prostate cancer cells (PC-3) more strongly than dacarbazine (DTIC), and the IC $\mathrm{C}_{50}$ value of $\mathbf{8 1}$ is lower than that of 5-fluorouracil with $13.5 \mu \mathrm{mol} / \mathrm{L}$.

Keywords triazene; 1,3,4-thiadiazole; human esophageal cancer cells; human gastric cancer cells; human prostate cancer cells
\end{abstract}

三氮烯类化合物是指一类含有 $\mathrm{N}=\mathrm{N}-\mathrm{N}$ 结构的化 合物, 又称重氮氨基化合物. 作为多电子中心, 可以和 Lewis 酸作用发生 Stille 偶联反应 ${ }^{[1]}$, 环合成特殊杂环 ${ }^{[2]}$. 此外, 三氮烯类化合物对癌细胞还表现出较强的抑制作 用, 如达卡巴嗪(DTIC)和替莫唑胺(TMZ) 是早已应用于<smiles>CN(C)/N=N/c1[nH]cnc1C(N)=O</smiles><smiles>CC(=O)c1ncn2c(=O)n(C)nnc12</smiles>

* Corresponding author. E-mail: longyue@zzu.edu.cn

Received September 3, 2018; revised October 27, 2018; published online December 21, 2018.

Project supported by the National Natural Science Foundation of China (No. J1210060), the Science and Technology Planning Project of Henan Province (No. 0624420031) and the Basic Research Development Program of Henan Province (No. 022463001).

国家自然科学基金(No. J1210060)、河南省科技攻关计划(No. 0624420031)和河南省基础研究基金(No. 022463001)资助项目. 
临床的三氮烯抗癌药物 ${ }^{[3]}$. 我们课题组也将三氮烯结构 分别和 1,3,4-噁二唑、含糖基的查尔酮结构相拼接合成 出两种三氮烯类化合物, 并对人胃癌细胞(MGC803)和 人前列腺癌细胞(PC-3)做活性篎选, 发现多个抑制作用 强于对照药物达卡巴嗪的新型三氮烯类化合物 ${ }^{[4,5]}$. 研 究结果表明, 三氮烯类化合物抗癌作用机理 ${ }^{[6]}$ 是含 $\mathrm{N}-$ $\mathrm{CH}_{3}$ 结构的三氮烯脱氮变为高活性甲基碳正离子, 并通 过该离子作用于 DNA 碱基片段, 干扰细胞分裂.

1,3,4-噻二唑是结构中含有两个氮原子和一个硫原 子的 “碳一硫-氮” 系列芳杂环. 近年来, 随着对该类化 合物研究的不断深入, 发现含 1,3,4-噻二唑结构的化合 物对癌细胞表现出较强的抑制作用 ${ }^{[7 \sim 11]}$. 李莎等 ${ }^{[12]}$ 将 1,3,4-噻二唑和二硫键拼接合成一系列 1,3,4-噻二唑二硫 键衍生物, 并通过抗癌活性篎选, 发现个别化合物对人 体肝癌细胞、乳腺癌细胞和肺癌细胞的抑制效果要优于 对照药 5-氟尿嘧啶. Juszczak 等 ${ }^{[13]}$ 通过研究 2-(4-氟苯胺 基)-5-(2,4-二羟基苯基)-1,3,4-噻二唑(FABT)对 A549 肺 癌细胞的生长抑制, 发现该类化合物通过抑制细胞外调 节蛋白激酶(ERK1/2)而导致细胞增殖停留在 $\mathrm{G} 0 / \mathrm{G} 1$ 期, 证实 2-氨基-1,3,4-噻二唑化合物具有一定的抗肿瘤生物 活性. 噻二唑环独特的富电性结构使得该类衍生物与生 物系统中的酶和生物受体能够很容易结合, 而且硫氮杂 环容易和金属离子螯合, 增强化合物的细胞通透性, 因 此可以表现出广泛的生物活性.

根据生物活性叠加原理, 将 1,3,4-噻二唑环和三氮 烯结构拼接, 合成了 15 个新型的 1,3,4-噻二唑三氮烯化 合物，并测试了该类化合物对人食管癌细胞 $(\mathrm{EC} 109)$ 、人 胃癌细胞(MGC803)和人前列腺癌细胞(PC-3)的抑制作 用.

\section{1 结果与讨论}

\section{1 合成}

在参考文献[14]的基础上，尝试用 1,3-二芳基硫脲 和取代基苯甲酰肼在三乙胺作催化剂, 水作溶剂条件 下, 加热回流反应 $3 \mathrm{~h}$ 后分离得到 1,3,4-噻二唑三氮烯类 化合物. 相比之下，中间体 1,3-二芳基硫脲的合成比文 献中二硫代酯更容易, 后处理更简便. 与文献结果相类 似，该反应过程中无副产物 1,3,4-噁二唑类化合物生成， 1,3-二芳基硫脲和取代基苯甲酰肼反应是合成 2-芳胺 基-1,3,4-噻二唑的一种新方法. 在进一步研究该反应的 机理中, 我们合成了中间体 7p , 再和 4-(3,3-二甲基三氮 烯-1-基)苯甲酰肼(5a) 在相同条件下进行反应, $3 \mathrm{~h}$ 后分 离得到 2-苯胺基-5-[4-(3,3-二甲基三氮烯-1-基)苯 基]-1,3,4-噻二唑(8a)和 $N$-甲基苯胺(Scheme 2), 当中间 体无 $\mathrm{N}-\mathrm{H}$ 时, 反应不能进行. 我们通过异硫氧酸苯酯

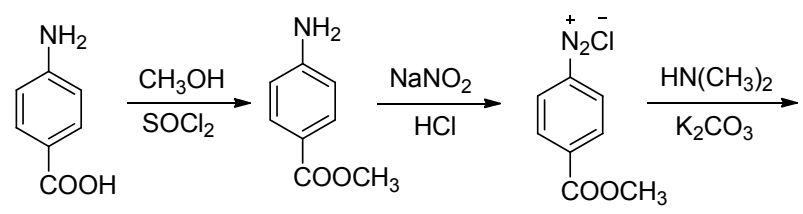

$1 a$

$2 a$

3a

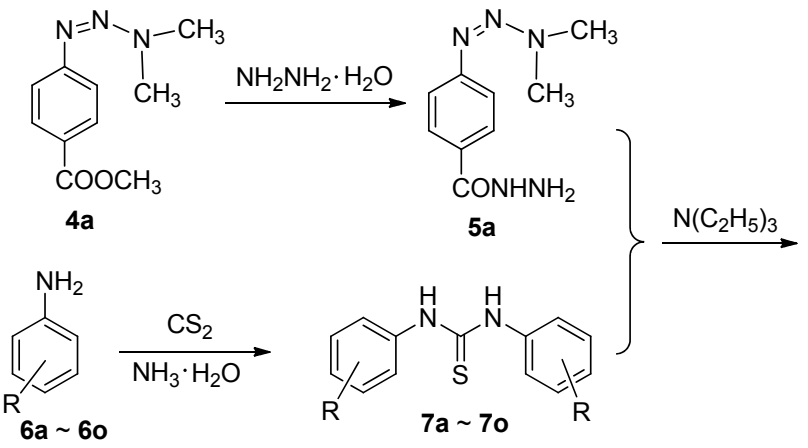<smiles>[R]c1ccc(Nc2nnc(-c3ccc(/N=N/N(C)C)cc3)s2)cc1</smiles>
8a: $\mathrm{R}=\mathrm{H}$;
8b: $\mathrm{R}=2-\mathrm{CH}_{3}$;
8c: $\mathrm{R}=3-\mathrm{CH}_{3}$
8d: $\mathrm{R}=4-\mathrm{CH}_{3} ; \quad$ 8e: $\mathrm{R}=2,6-\left(\mathrm{CH}_{3}\right)_{2} ; \quad$ 8f: $\mathrm{R}=2-\mathrm{OCH}_{3}$
8g: $\mathrm{R}=3-\mathrm{OCH}_{3} ; \quad 8 \mathbf{~ h : ~} \mathrm{R}=4-\mathrm{OCH}_{3} ; \quad$ 8i: $\mathrm{R}=2-\mathrm{Cl}$
8j: $\mathrm{R}=3-\mathrm{Cl} ; \quad \mathbf{8 k}: \mathrm{R}=4-\mathrm{Cl}$
$8 \mathrm{~m}: \mathrm{R}=2-\mathrm{Br} ; \quad 8 \mathrm{n}: \mathrm{R}=4-\mathrm{Br}$
8I: $\mathrm{R}=3,4-\mathrm{Cl}_{2}$
80: $R=4-F$

图式 1 1,3,4-噻二唑三氮烯类化合物的合成

Scheme 1 Synthesis of 1,3,4-thiadiazole triazene compounds

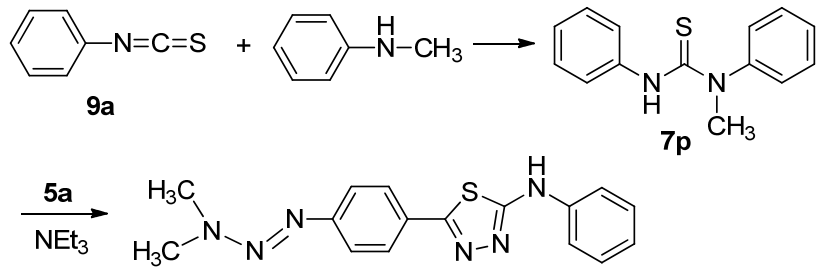

8a

图式 2 化合物 $7 \mathrm{p}$ 和 $5 \mathrm{a}$ 的反应

Scheme 2 Reaction of compounds $7 \mathbf{p}$ and $5 \mathbf{a}$<smiles>CCN(C)CCN(C)N=Nc1ccc(C(=O)NN)cc1</smiles><smiles>CN(C)N=Nc1ccc(-c2nnc(Nc3ccccc3)s2)cc1</smiles>

(9a)和化合物 $5 \mathbf{a}$ 在相同条件下进行反应, $3 \mathrm{~h}$ 后分离得到 了化合物 8a (Eq. 1), 由此推出的反应机理如 Scheme 3 所示.

\section{2 化合物的抗肿瘤活性}

我们选用三氮烯药物达卡巴嗪(DTIC)和 5-氟尿嘧 
啶 (5-FU) 作对照, 测试该类化合物对人食管癌细胞 (EC109)、人胃癌细胞 (MGC803) 和人前列腺癌细胞 (PC-3)三种癌细胞的抑制作用. 其 $\mathrm{IC}_{50}$ 值见表 1 .
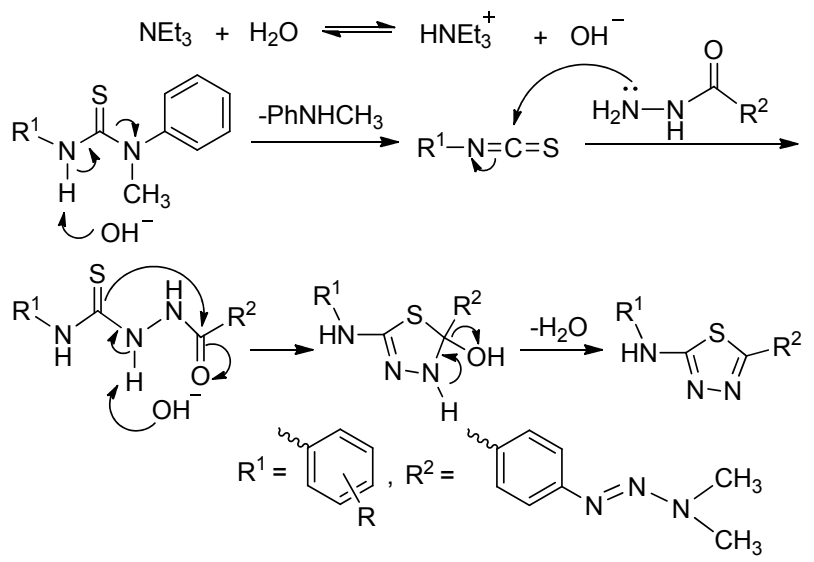

图式 3 1,3,4-噻二唑的反应机理

Scheme 3 Reaction mechanism of 1,3,4-thiadiazole

表 1 1,3,4-噻二唑三氮烯类化合物的抗癌活性 $\left[\mathrm{IC}_{50} /(\mu \mathrm{mol}\right.$ $\left.\left.\mathrm{L}^{-1}\right)\right]$

Table 1 Anticancer activity of 1,3,4-thiadiazole triazene compounds $\left[\mathrm{IC}_{50} /\left(\mu \mathrm{mol} \cdot \mathrm{L}^{-1}\right)\right]$

\begin{tabular}{clll}
\hline Compd. & \multicolumn{1}{c}{ EC109 } & \multicolumn{1}{c}{ MGC803 } & \multicolumn{1}{c}{ PC-3 } \\
\hline $\mathbf{8 a}$ & $161.1 \pm 3.7$ & $37.0 \pm 6.2$ & $94.5 \pm 0.6$ \\
$\mathbf{8 b}$ & $>300.0$ & $59.6 \pm 9.6$ & $232.4 \pm 7.0$ \\
$\mathbf{8 c}$ & $>300.0$ & $5.3 \pm 0.3$ & $>300.0$ \\
$\mathbf{8 d}$ & $128.2 \pm 7.9$ & $113.3 \pm 5.5$ & $95.8 \pm 0.1$ \\
$\mathbf{8 e}$ & $191.2 \pm 1.9$ & $99.3 \pm 1.1$ & $119.9 \pm 3.4$ \\
$\mathbf{8 f}$ & $208.8 \pm 4.0$ & $6.5 \pm 0.7$ & $65.3 \pm 4.6$ \\
$\mathbf{8 g}$ & $>300.0$ & $42.3 \pm 2.1$ & $109.8 \pm 4.6$ \\
$\mathbf{8 h}$ & $161.1 \pm 2.3$ & $94.7 \pm 0.3$ & $73.8 \pm 1.4$ \\
$\mathbf{8 i}$ & $73.7 \pm 6.0$ & $48.2 \pm 8.4$ & $47.6 \pm 2.3$ \\
$\mathbf{8 j}$ & $>300.0$ & $45.4 \pm 0.5$ & $30.6 \pm 0.5$ \\
$\mathbf{8 k}$ & $218.4 \pm 4.3$ & $12.2 \pm 2.0$ & $113.3 \pm 0.6$ \\
$\mathbf{8 1}$ & $113.8 \pm 1.9$ & $6.3 \pm 0.4$ & $13.5 \pm 2.1$ \\
$\mathbf{8 m}$ & $166.5 \pm 0.8$ & $49.1 \pm 2.7$ & $127.3 \pm 6.2$ \\
$\mathbf{8 n}$ & $187.5 \pm 1.3$ & $9.6 \pm 0.3$ & $59.0 \pm 3.3$ \\
$\mathbf{8 0}$ & $>300.0$ & $109.7 \pm 9.1$ & $72.3 \pm 5.3$ \\
DTIC & $108.1 \pm 1.2$ & $99.6 \pm 4.1$ & $146.4 \pm 5.1$ \\
5 -FU & $10.3 \pm 0.8$ & $7.2 \pm 1.2$ & $25.3 \pm 1.8$ \\
\hline
\end{tabular}

从表 1 可以看出, 大部分化合物对人食管癌细胞 (EC109)、人胃癌细胞 (MGC803) 和人前列腺癌细胞 (PC-3) 具有较强的抑制作用, 而且与药物达卡巴嗪 (DTIC) 相比, 化合物 $8 \mathrm{a} \sim 8 \mathrm{c} 、 8 \mathrm{e} \sim 8 \mathrm{n}$ 对人胃癌细胞 (MGC803)抑制作用和化合物 8a、8d $\sim 80$ 对人前列腺癌 细胞(PC-3)抑制作用都强于达卡巴嗪(DTIC), 其中化合 物 $8 \mathrm{i}$ 对三种癌细胞的 $\mathrm{IC}_{50}$ 值都低于达卡巴嗪(DTIC), 和 经典抗癌药物 5-氟尿嘧啶(5-FU)对比，化合物 8c、8f、 81 对人胃癌细胞(MGC803)的抑制作用和化合物 81 对人 前列腺癌细胞(PC-3)的抑制作用更好一些. 从检测结果
对比来看，三种癌细胞的活性抑制受化合物取代基的影 响较大. 在人食管癌细胞(EC109)检测中, 三种间位取 代基均表现出较小的抑制作用. 对位取代基的活性比 较, 有如下顺序: $\mathrm{CH}_{3}>\mathrm{OCH}_{3} \approx \mathrm{H}>\mathrm{Br}>\mathrm{Cl}>\mathrm{F}$, 部分带 供电子基的该类化合物对人食管癌细胞(EC109)抑制相 对明显; 在人胃癌细胞(MGC803)检测中，对位上 $\mathrm{Br}$ 、 $\mathrm{Cl}$ 取代化合物表现出很强的抑制活性，大多数邻、间位 取代化合物表现出比达卡巴嗪(DTIC)强许多的药物活 性; 在人前列腺癌细胞(PC-3)检测中，对位取代基化合 物全部表现出比达卡巴嗪(DTIC)更强的药物活性, 除甲 基外，其他取代基在邻、间位同样表现出较强的抑制活 性，其中 3,4-二氯取代(81) 的活性可以达到达卡巴嗪 (DTIC)的 10 倍左右.

\section{2 结论}

将 1,3,4-噻二唑环和三氮烯结构拼接, 合成了 15 个 新型的三氮烯化合物，该类化合物的结构都通过核磁共 振波谱(NMR)、红外光谱(IR)和高分辨质谱(HRMS)等方 法得到了验证，并对其抗癌活性做出活性筛选，结果证 明化合物 8a $\sim 8 \mathrm{c}, 8 \mathrm{e} \sim \mathbf{8 n}$ 对人胃癌细胞( MGC803) 抑制 作用和化合物 8a, 8d $\sim 80$ 对人前列腺癌细胞( $\mathrm{PC}-3$ )抑制 作用都强于对照药物达卡巴嗪(DTIC)，其中化合物 8i 对 三种癌细胞的 $\mathrm{IC}_{50}$ 值都低于达卡巴嗪(DTIC), 化合物 8c、8f、81 对人胃癌细胞(MGC803)的抑制作用和化合物 81 对人前列腺癌细胞 (PC-3)的抑制作用比药物 5-氟尿嘧 啶(5-FU)还要强一些.

\section{3 实验部分}

\section{1 仪器与试剂}

ZF7 三用紫外分析仪(巩义市予华仪器有限责任有 限公司); 84-1 磁力搅拌控温电热套(山东甄成华鲁电热 仪器有限公司); SHB-III 循环水式多用真空原(郑州长城 科工贸有限公司); CP214 电子天平(奥豪斯仪器有限公 司); Q-TOF Micro 高分辨质谱测定仪(美国 Waters 公司); RE5299 旋转蒸发仪 (上海亚荣生化仪器厂); DPX-400FT 超导核磁共振仪(美国 Bruker 公司); Vector 22 傅里叶变 换红外光谱仪(美国 Bruker 公司). 所用试剂均为市售分 析纯，使用前按常规方法处理. 实验所用的肿瘤细胞: 人食管癌细胞 $(E C 109) 、 人$ 胃癌细胞( MGC 803$)$ 和人前列 腺癌细胞(PC-3)由郑州大学基础医学院药理系提供.

\section{2 化合物的合成}

\subsubsection{4-氨基苯甲酸甲酯(2a)的合成}

化合物 2a 合成方法参考文献[15]. 在 $100 \mathrm{~mL}$ 圆底 烧瓶中加入 $1.00 \mathrm{~g}(7.29 \mathrm{mmol})$ 对氨基苯甲酸, $1.00 \mathrm{~g}$ (8.41 mmol)氯化亚砜和 $30 \mathrm{~mL}$ 甲醇, 升温回流 $2 \mathrm{~h}$, 减压 
旋干溶剂, 加入少量稀氨水, 用乙酸乙酯萃取, 旋干有 机相, 得对氨基苯甲酸甲酯 (2a), 产率 93.8\%, m.p. $112 \sim 113{ }^{\circ} \mathrm{C}^{[16]}$.

3.2.24-(3,3-二甲基三氮烯-1-基)苯甲酸甲酯(4a)的 合成

化合物 4a 合成方法参考文献[15]. 在 $100 \mathrm{~mL}$ 圆底 烧瓶中将 $1.10 \mathrm{~g}$ (7.28 mmol)化合物 $2 \mathbf{a}$ 溶于 $15 \mathrm{~mL} 10 \%$ 的盐酸中, 冰水浴搅拌. 用少量水将 $1.00 \mathrm{~g}(14.49$ $\mathrm{mmol}$ )亚硝酸钠溶解, 滴加到反应瓶, 继续摚拌 $1 \mathrm{~h}$, 得 到重氮盐. 在 $250 \mathrm{~mL}$ 圆底烧瓶中加入 $2.90 \mathrm{~g}(21.22$ $\mathrm{mmol}) 33 \%$ 二甲胺和 $3.00 \mathrm{~g}(21.71 \mathrm{mmol})$ 碳酸钾, 再加 入重氮盐, 搅拌 $30 \mathrm{~min}$ 后抽滤得化合物 $\mathbf{4 a}$, 产率 $96.6 \%$, $99 \sim 100{ }^{\circ} \mathrm{C}^{[17]}$.

3.2.34-(3,3-二甲基三氮烯-1-基)苯甲酰肼(5a)的合 成

化合物 5a 合成方法参考文献[15]. 在 $100 \mathrm{~mL}$ 圆底 烧瓶中将 $0.61 \mathrm{~g}(2.97 \mathrm{mmol})$ 化合物 $4 \mathbf{a}$ 和 $1.10 \mathrm{~g}$ (17.60 mmol) $80 \%$ 水合肼溶于适量甲醇中, 加热回流 $4 \mathrm{~h}$, 旋蒸 出大量溶剂后加水抽滤, 得化合物 5a. 产率 83.8\%, m.p. $155 \sim 156{ }^{\circ} \mathrm{C}^{[18]}$.

\section{2 .4 1,3-二芳基硫脲 $(7 \mathbf{a} \sim 70)$ 的合成}

在 $50 \mathrm{~mL}$ 圆底烧瓶中加入 $20.00 \mathrm{mmol}$ 化合物 $\mathbf{6 a} \sim$ 6o, $0.77 \mathrm{~g}(10.11 \mathrm{mmol})$ 二硫化碳, $5 \mathrm{~mL}$ 浓氨水和 $10 \mathrm{~mL}$ 水, 常温搅拌 $8 \mathrm{~h}$, 有大量固体析出, 抽滤, 水洗, 干燥, 得化合物 7a $\sim 70$.

1,3-二(2-溴苯基)硫脲 $(7 \mathbf{m})$ : 白色固体, 产率 $87.9 \%$. m.p. $157 \sim 158{ }^{\circ} \mathrm{C}$; ${ }^{1} \mathrm{H}$ NMR (DMSO- $\left.d_{6}, 600 \mathrm{MHz}\right) \delta$ : $9.59(\mathrm{~s}, 2 \mathrm{H}), 7.65 \sim 7.69(\mathrm{~m}, 4 \mathrm{H}), 7.40(\mathrm{t}, J=7.8 \mathrm{~Hz}, 2 \mathrm{H})$, $7.20(\mathrm{t}, J=7.9 \mathrm{~Hz}, 2 \mathrm{H}) ;{ }^{13} \mathrm{C}$ NMR (DMSO- $\left.d_{6}, 151 \mathrm{MHz}\right)$ $\delta: 181.25,137.56,132.53,130.08,127.96,127.73,120.85$. 其余化合物参考文献[19２2].

\subsubsection{2-芳胺基-5-[4-(3,3-二甲基三氮烯-1-基)苯} 基]-1,3,4-噻二唑 $(\mathbf{8 a} \sim \mathbf{8 0})$ 的合成

在 $100 \mathrm{~mL}$ 圆底烧瓶中加入 $0.50 \mathrm{~g}(2.42 \mathrm{mmol})$ 化合 物 5a, $2.20 \mathrm{mmol}$ 化合物 7a $7 \mathbf{7}$ 和 $0.50 \mathrm{~g}(4.95 \mathrm{mmol})$ 三 乙胺, 水 $40 \mathrm{~mL}$, 加热回流 $3 \mathrm{~h}$ 后, 冷却, 用乙酸乙酯萃 取, 柱层析分离 [洗脱剂为乙酸乙酯一石油醚 $(V: V=1:$ $6)$ ], 得目标产物 $\mathbf{8 a} \sim \mathbf{8 0}$.

2-苯胺基-5-[4-(3,3-二甲基三氮烯-1-基)苯基]-1,3,4噻二唑 $(8 \mathrm{a})$ : 淡黄色固体，产率 87.4\%. m.p. 243 $245{ }^{\circ} \mathrm{C} ;{ }^{1} \mathrm{H}$ NMR (DMSO- $\left.d_{6}, 600 \mathrm{MHz}\right) \delta: 14.08$ (s, 1H), 7.50 (d, $J=5.6 \mathrm{~Hz}, 3 \mathrm{H}), 7.36$ (d, $J=3.7 \mathrm{~Hz}, 2 \mathrm{H}), 7.24$ (d, $J=2.6 \mathrm{~Hz}, 4 \mathrm{H}), 3.47(\mathrm{~s}, 3 \mathrm{H}), 3.12(\mathrm{~s}, 3 \mathrm{H}) ;{ }^{13} \mathrm{C} \mathrm{NMR}$ $\left(\mathrm{DMSO}-d_{6}, 151 \mathrm{MHz}\right) \delta$ : $168.94,152.24,150.95,135.16$, $129.82,129.78,129.43,129.19,122.33,120.39,43.42$,
36.39; IR v: 2919, 2851, 2737, 1599, 1495, 1323, 1080, 846, 702, $612 \mathrm{~cm}^{-1}$; HRMS calcd for $\mathrm{C}_{16} \mathrm{H}_{16} \mathrm{~N}_{6} \mathrm{SNa}$ $[\mathrm{M}+\mathrm{Na}]^{+}$347.1055, found 347.1054.

2-(2-甲基苯胺基)-5-[4-(3,3-二甲基三氮烯-1-基)苯 基]-1,3,4-噻二唑 $(\mathbf{8 b})$ : 淡黄色固体, 产率 77.7\%. m.p. $242 \sim 244{ }^{\circ} \mathrm{C} ;{ }^{1} \mathrm{H}$ NMR (DMSO- $\left.d_{6}, 600 \mathrm{MHz}\right) \delta: 14.10$ (s, $1 \mathrm{H}), 7.43$ (t, $J=7.5 \mathrm{~Hz}, 1 \mathrm{H}), 7.40$ (d, $J=7.6 \mathrm{~Hz}, 1 \mathrm{H}), 7.35$ $(\mathrm{t}, J=7.5 \mathrm{~Hz}, 1 \mathrm{H}), 7.31(\mathrm{~d}, J=7.8 \mathrm{~Hz}, 1 \mathrm{H}), 7.23(\mathrm{~d}, J=2.5$ $\mathrm{Hz}, 4 \mathrm{H}), 3.46$ (s, 3H), 3.12 (s, 3H), 2.02 (s, 3H); ${ }^{13} \mathrm{C} \mathrm{NMR}$ (DMSO- $\left.d_{6}, 151 \mathrm{MHz}\right) \delta: 168.57,152.38,150.71,136.38$, $134.29,131.57,130.44,129.77,128.69,127.70,122.26$, 120.54, 43.40, 36.38, 17.77; IR v: 3083, 2919, 2744, 1603, 1510, 1315, 1086, 996, 842, $727 \mathrm{~cm}^{-1}$; HRMS calcd for $\mathrm{C}_{17} \mathrm{H}_{19} \mathrm{~N}_{6} \mathrm{~S}[\mathrm{M}+\mathrm{H}]^{+}$339.1392, found 339.1390.

2-(3-甲基苯胺基)-5-[4-(3,3-二甲基三氮烯-1-基)苯 基]-1,3,4-噻二唑 $(8 \mathrm{c})$ ：淡黄色固体，产率 89.0\%. m.p. $255 \sim 257{ }^{\circ} \mathrm{C} ;{ }^{1} \mathrm{H}$ NMR (DMSO- $\left.d_{6}, 600 \mathrm{MHz}\right) \delta: 14.06$ (s, $1 \mathrm{H}), 7.38(\mathrm{dd}, J=7.8,2.3 \mathrm{~Hz}, 1 \mathrm{H}), 7.31(\mathrm{~d}, J=7.7 \mathrm{~Hz}$, $1 \mathrm{H}), 7.25$ (d, $J=2.3 \mathrm{~Hz}, 4 \mathrm{H}), 7.19$ (s, 1H), 7.13 (d, $J=7.9$ $\mathrm{Hz}, 1 \mathrm{H}), 3.48$ (s, 3H), 3.13 (s, 3H), 2.32 (s, 3H); ${ }^{13} \mathrm{C}$ NMR (DMSO- $\left.d_{6}, 151 \mathrm{MHz}\right) \delta: 168.97,152.23,150.88,139.39$, $135.10,130.52,129.58,129.46,129.34,126.25,122.36$, 120.39, 43.41, 36.40, 21.22; IR v: 2957, 2918, 2851, 2735, 1729, 1603, 1488, 1355, 1102, $709 \mathrm{~cm}^{-1}$; HRMS calcd for $\mathrm{C}_{17} \mathrm{H}_{18} \mathrm{~N}_{6} \mathrm{SNa}[\mathrm{M}+\mathrm{Na}]^{+}$361.1212, found 361.1209.

2-(4-甲基苯胺基)-5-[4-(3,3-二甲基三氮烯-1-基)苯 基]-1,3,4-噻二唑(8d)：淡黄色固体，产率 80.2\%. m.p. $237 \sim 239{ }^{\circ} \mathrm{C} ;{ }^{1} \mathrm{H}$ NMR (DMSO- $\left.d_{6}, 600 \mathrm{MHz}\right) \delta: 14.04$ (s, $1 \mathrm{H}), 7.29$ (d, $J=8.0 \mathrm{~Hz}, 2 \mathrm{H}), 7.24 \sim 7.26(\mathrm{~m}, 4 \mathrm{H}), 7.21(\mathrm{~d}$, $J=8.0 \mathrm{~Hz}, 2 \mathrm{H}), 3.48$ (s, 3H), 3.13 (s, 3H), 2.36 (s, 3H); ${ }^{13} \mathrm{C}$ NMR (DMSO- $\left.d_{6}, 151 \mathrm{MHz}\right) \delta: 169.02,152.20$, $151.01,139.38,132.57,130.27,129.44,128.87,122.41$, 120.40, 43.40, 36.38, 21.25; IR v: 3088, 2919, 2851, 1604, $1507,1376,1323,1167,1073,840 \mathrm{~cm}^{-1}$; HRMS calcd for $\mathrm{C}_{17} \mathrm{H}_{18} \mathrm{~N}_{6} \mathrm{SNa}[\mathrm{M}+\mathrm{Na}]^{+}$361.1212, found 361.1210.

2-(2,6-二甲基苯胺基)-5-[4-(3,3-二甲基三氮烯-1基)苯基]-1,3,4-噻二唑(8e)：白色固体，产率 50.9\%. m.p. $231 \sim 233{ }^{\circ} \mathrm{C} ;{ }^{1} \mathrm{H}$ NMR (DMSO- $\left.d_{6}, 400 \mathrm{MHz}\right) \delta$ : 14.21 (s, $1 \mathrm{H}), 7.35(\mathrm{t}, J=7.5 \mathrm{~Hz}, 1 \mathrm{H}), 7.24(\mathrm{dd}, J=8.2,5.3 \mathrm{~Hz}$, $6 \mathrm{H}), 3.47(\mathrm{~s}, 3 \mathrm{H}), 3.12(\mathrm{~s}, 3 \mathrm{H}), 1.97(\mathrm{~s}, 6 \mathrm{H}) ;{ }^{13} \mathrm{C} \mathrm{NMR}$ $\left(\mathrm{CDCl}_{3}, 151 \mathrm{MHz}\right) \delta: 167.91,152.67,150.87,136.48$, $132.86,130.24,129.03,127.63,121.91,120.78,43.21$, 36.01, 18.12; IR v: 3087, 2917, 2741, 1605, 1509, 1321, $1159,1094,965,783 \mathrm{~cm}^{-1}$; HRMS calcd for $\mathrm{C}_{18} \mathrm{H}_{20} \mathrm{~N}_{6} \mathrm{SNa}$ $[\mathrm{M}+\mathrm{Na}]^{+}$375.1368, found 375.1366 . 
2-(2-甲氧基苯胺基)-5-[4-(3,3-二甲基三氮烯-1-基) 苯基]-1,3,4-噻二唑 $(8 \mathbf{8})$ : 淡黄色固体, 产率 77.9\%. m.p. $233 \sim 235{ }^{\circ} \mathrm{C} ;{ }^{1} \mathrm{H}$ NMR (DMSO- $\left.d_{6}, 600 \mathrm{MHz}\right) \delta: 14.02$ (s, $1 \mathrm{H}), 7.49$ (t, $J=8.1 \mathrm{~Hz}, 1 \mathrm{H}), 7.42(\mathrm{~d}, J=7.7 \mathrm{~Hz}, 1 \mathrm{H})$, $7.24 \sim 7.27(\mathrm{~m}, 4 \mathrm{H}), 7.15(\mathrm{dd}, J=8.5,2.7 \mathrm{~Hz}, 1 \mathrm{H}), 7.11$ (dd, $J=7.7,2.6 \mathrm{~Hz}, 1 \mathrm{H}), 3.56$ (s, 3H), 3.47 (s, 3H), 3.12 (s, $3 \mathrm{H}$ ); ${ }^{13} \mathrm{C}$ NMR (DMSO- $\left.d_{6}, 151 \mathrm{MHz}\right) \delta: 169.26,154.97$, $151.43,131.78,130.92,128.50,123.67,122.73,121.35$, 120.39, 113.46, 56.27, 43.38, 36.36; IR $v: 3060,2901$, 2754, 1603, 1506, 1399, 1288, 1161, 1044, $852 \mathrm{~cm}^{-1}$; HRMS calcd for $\mathrm{C}_{17} \mathrm{H}_{18} \mathrm{~N}_{6} \mathrm{OSNa}[\mathrm{M}+\mathrm{Na}]^{+} 377.1161$, found 377.1158 .

2-(3-甲氧基苯胺基)-5-[4-(3,3-二甲基三氮烯-1-基) 苯基]-1,3,4-噻二唑 $(8 \mathrm{~g})$ : 橙黄色固体, 产率 79.4\%. m.p. $235 \sim 237{ }^{\circ} \mathrm{C} ;{ }^{1} \mathrm{H}$ NMR (DMSO- $\left.d_{6}, 600 \mathrm{MHz}\right) \delta: 14.07$ (s, $1 \mathrm{H}), 7.40$ (td, $J=8.1,2.7 \mathrm{~Hz}, 1 \mathrm{H}), 7.23 \sim 7.31(\mathrm{~m}, 4 \mathrm{H})$, 7.07 (d, $J=8.1 \mathrm{~Hz}, 1 \mathrm{H}), 7.00$ (s, 1H), 6.89 (d, $J=7.8 \mathrm{~Hz}$, $1 \mathrm{H}), 3.74(\mathrm{~s}, 3 \mathrm{H}), 3.48(\mathrm{~s}, 3 \mathrm{H}), 3.13(\mathrm{~s}, 3 \mathrm{H}) ;{ }^{13} \mathrm{C} \mathrm{NMR}$ (DMSO- $\left.d_{6}, 151 \mathrm{MHz}\right) \delta: 168.90,160.13,152.25,150.93$, 136.16, 130.54, 129.37, 122.39, 121.23, 120.39, 115.32, 115.28, 55.93, 43.40, 36.37; IR v: 3067, 2921, 2751, 1604, $1490,1332,1215,1158,1046,855 \mathrm{~cm}^{-1}$; HRMS calcd for $\mathrm{C}_{17} \mathrm{H}_{18} \mathrm{~N}_{6} \mathrm{OSNa}[\mathrm{M}+\mathrm{Na}]^{+}$377.1161, found 377.1159.

2-(4-甲氧基苯胺基)-5-[4-(3,3-二甲基三氮烯-1-基) 苯基]-1,3,4-噻二唑 $(\mathbf{8 h})$ : 淡黄色固体, 产率 87.0\%. m.p. $230 \sim 231{ }^{\circ} \mathrm{C} ;{ }^{1} \mathrm{H}$ NMR (DMSO- $d_{6}, 600 \mathrm{MHz}$ ) $\delta: 14.03$ (s, $1 \mathrm{H}), 7.26 \sim 7.27(\mathrm{~m}, 6 \mathrm{H}), 7.02 \sim 7.06(\mathrm{~m}, 2 \mathrm{H}), 3.81(\mathrm{~s}$, $3 \mathrm{H}), 3.48$ (s, 3H), 3.13 (s, $3 \mathrm{H}$ ); ${ }^{13} \mathrm{C}$ NMR (DMSO- $d_{6}, 151$ MHz) $\delta: 169.22,159.99,152.20,151.11,130.34,129.40$, 127.70, 122.48, 120.41, 114.93, 55.88, 43.38, 36.36; IR v: 3108, 2926, 2748, 1732, 1603, 1508, 1254, 1097, 1034, $852 \mathrm{~cm}^{-1}$; HRMS calcd for $\mathrm{C}_{17} \mathrm{H}_{18} \mathrm{~N}_{6} \mathrm{OSNa}[\mathrm{M}+\mathrm{Na}]^{+}$ 377.1161, found 377.1159 .

2-(2-氯苯胺基)-5-[4-(3,3-二甲基三氮烯-1-基)苯 基]-1,3,4-噻二唑(8i)：白色固体, 产率 79.0\%. m.p. 227 $228{ }^{\circ} \mathrm{C} ;{ }^{1} \mathrm{H}$ NMR (DMSO- $\left.d_{6}, 600 \mathrm{MHz}\right) \delta: 14.13(\mathrm{~s}, 1 \mathrm{H}$ ), $7.65 \sim 7.70(\mathrm{~m}, 2 \mathrm{H}), 7.54 \sim 7.59(\mathrm{~m}, 2 \mathrm{H}), 7.24 \sim 7.29(\mathrm{~m}$, $4 \mathrm{H}), 3.47$ (s, 3H), 3.12 (s, $3 \mathrm{H}) ;{ }^{13} \mathrm{C}$ NMR (DMSO- $d_{6}, 151$ MHz) $\delta: 169.08,152.51,150.77,132.77,132.33,132.31$, $132.23,130.75,128.97,128.71,122.11,120.59,43.42$, 36.40; IR v: 3063, 2901, 2748, 1736, 1602, 1477, 1329, $1075,968,843 \mathrm{~cm}^{-1}$; HRMS calcd for $\mathrm{C}_{16} \mathrm{H}_{15} \mathrm{ClN}_{6} \mathrm{SNa}$ $[\mathrm{M}+\mathrm{Na}]^{+}$381.0665, found 381.0662.

2-(3-氯苯胺基)-5-[4-(3,3-二甲基三氮烯-1-基)苯 基]-1,3,4-噻二唑 $(8 \mathbf{j})$ : 白色固体, 产率 77.4\%. m.p. 232
$233{ }^{\circ} \mathrm{C} ;{ }^{1} \mathrm{H}$ NMR (DMSO- $\left.d_{6}, 600 \mathrm{MHz}\right) \delta: 14.12(\mathrm{~s}, 1 \mathrm{H})$, 7.60 (t, $J=1.8 \mathrm{~Hz}, 1 \mathrm{H}), 7.57 \sim 7.58(\mathrm{~m}, 1 \mathrm{H}), 7.51$ (d, $J=$ $8.0 \mathrm{~Hz}, 1 \mathrm{H}), 7.33 \sim 7.34(\mathrm{~m}, 1 \mathrm{H}), 7.25 \sim 7.28(\mathrm{~m}, 4 \mathrm{H}), 3.48$ (s, 3H), 3.13 (s, 3H); ${ }^{13} \mathrm{C}$ NMR (DMSO- $\left.d_{6}, 151 \mathrm{MHz}\right) \delta$ : $168.89,152.34,150.84,136.46,133.73,131.29,129.96$, $129.57,129.41,128.20,122.14,120.46,43.41,36.39$; IR $v$ : 3079, 2902, 2748, 1604, 1508, 1481, 1314, 1093, 849, 711 $\mathrm{cm}^{-1}$; HRMS calcd for $\mathrm{C}_{16} \mathrm{H}_{15} \mathrm{ClN}_{6} \mathrm{SNa}[\mathrm{M}+\mathrm{Na}]^{+}$ 381.0665 , found 381.0664 .

2-(4-氯苯胺基)-5-[4-(3,3-二甲基三氮烯-1-基)苯 基]-1,3,4-噻二唑 $(8 \mathbf{k})$ : 白色固体, 产率 87.7\%. m.p. $234 \sim 235{ }^{\circ} \mathrm{C} ;{ }^{1} \mathrm{H}$ NMR (DMSO- $\left.d_{6}, 600 \mathrm{MHz}\right) \delta: 14.13$ (s, $1 \mathrm{H}), 7.58(\mathrm{~d}, J=8.2 \mathrm{~Hz}, 2 \mathrm{H}), 7.42(\mathrm{~d}, J=8.3 \mathrm{~Hz}, 2 \mathrm{H})$, $7.26 \sim 7.29(\mathrm{~m}, 4 \mathrm{H}), 3.48(\mathrm{~s}, 3 \mathrm{H}), 3.13(\mathrm{~s}, 3 \mathrm{H}) ;{ }^{13} \mathrm{C} \mathrm{NMR}$ (DMSO- $\left.d_{6}, 151 \mathrm{MHz}\right) \delta: 168.89,152.33,150.91,134.44$, $134.05,131.14,129.83,129.59,122.17,120.48,43.41$, 36.40; IR v: 3084, 2921, 2794, 1740, 1603, 1494, 1323, 1238, 1090, $852 \mathrm{~cm}^{-1}$; HRMS calcd for $\mathrm{C}_{16} \mathrm{H}_{15} \mathrm{ClN}_{6} \mathrm{SNa}$ $[\mathrm{M}+\mathrm{Na}]^{+} 381.0665$, found 381.0661 .

2-(3,4-二氯苯胺基)-5-[4-(3,3-二甲基三氮烯-1-基) 苯基]-1,3,4-噻二唑 (81): 白色固体, 产率 79.7\%. m.p. $228 \sim 231{ }^{\circ} \mathrm{C} ;{ }^{1} \mathrm{H}$ NMR (DMSO- $\left.d_{6}, 600 \mathrm{MHz}\right) \delta: 14.16$ (s, 1H), 7.85 (s, 1H), 7.79 (dd, $J=8.6 \mathrm{~Hz}, 2.8 \mathrm{~Hz}, 1 \mathrm{H}), 7.41$ (dd, $J=8.6,3.0 \mathrm{~Hz}, 1 \mathrm{H}), 7.30(\mathrm{~d}, J=2.7 \mathrm{~Hz}, 4 \mathrm{H}), 3.49$ (s, $3 \mathrm{H}$ ), $3.14(\mathrm{~s}, 3 \mathrm{H}) ;{ }^{13} \mathrm{C}$ NMR (DMSO- $\left.d_{6}, 151 \mathrm{MHz}\right) \delta$ : 168.81, 152.39, 150.80, 134.99, 132.77, 131.95, 131.62, $131.57,129.82,129.68,121.99,120.53,43.42,36.40$; IR $v$ : 3087, 2922, 2751, 1757, 1508, 1476, 1328, 1092, 1005, $843 \mathrm{~cm}^{-1}$; HRMS calcd for $\mathrm{C}_{16} \mathrm{H}_{15} \mathrm{Cl}_{2} \mathrm{~N}_{6} \mathrm{~S}[\mathrm{M}+\mathrm{H}]^{+}$ 393.0456, found 393.0454.

2-(2-溴苯胺基)-5-[4-(3,3-二甲基三氮烯-1-基)苯 基]-1,3,4-噻二唑 $(8 \mathrm{~m})$ : 白色固体，产率 69.7\%. m.p. $219 \sim 221{ }^{\circ} \mathrm{C} ;{ }^{1} \mathrm{H}$ NMR (DMSO- $d_{6}, 600 \mathrm{MHz}$ ) $\delta: 14.14$ (s, $1 \mathrm{H}), 7.80(\mathrm{~d}, J=8.1,1 \mathrm{H}), 7.68$ (d, $J=7.8 \mathrm{~Hz}, 1 \mathrm{H}), 7.59$ (t, $J=7.7 \mathrm{~Hz}, 1 \mathrm{H}), 7.48(\mathrm{t}, J=7.7 \mathrm{~Hz}, 1 \mathrm{H}), 7.27(\mathrm{q}, J=8.5$ $\mathrm{Hz}, 4 \mathrm{H}$ ), 3.47 (s, 3H), 3.12 (s, 3H); ${ }^{13} \mathrm{C}$ NMR (DMSO- $d_{6}$, $151 \mathrm{MHz}) \delta$ : $168.94,152.47,150.60,134.37,133.90$, $132.37,132.33,129.53,128.77,123.06,122.18,120.56$, 43.43, 36.41; IR $v: 3084,2901,1737,1508,1476,1316$, $1162,1065,843,727 \mathrm{~cm}^{-1}$; HRMS calcd for $\mathrm{C}_{16} \mathrm{H}_{15} \mathrm{BrN}_{6} \mathrm{~S}-$ $\mathrm{Na}[\mathrm{M}+\mathrm{Na}]^{+}$425.0160, found 425.0159 .

2-(4-溴苯胺基)-5-[4-(3,3-二甲基三氮烯-1-基)苯 基]-1,3,4-噻二唑 $(\mathbf{8 n})$ : 淡黄色固体, 产率 79.5\%. m.p. $225 \sim 226{ }^{\circ} \mathrm{C} ;{ }^{1} \mathrm{H}$ NMR (DMSO- $\left.d_{6}, 600 \mathrm{MHz}\right) \delta: 14.12$ (s, $1 \mathrm{H}), 7.71(\mathrm{dd}, J=8.6,2.5 \mathrm{~Hz}, 2 \mathrm{H}), 7.35(\mathrm{~d}, J=8.7 \mathrm{~Hz}$, 
2H), $7.28(\mathrm{t}, J=6.6 \mathrm{~Hz}, 4 \mathrm{H}), 3.49(\mathrm{~s}, 3 \mathrm{H}), 3.14(\mathrm{~s}, 3 \mathrm{H}) ;{ }^{13} \mathrm{C}$ NMR (DMSO- $\left.d_{6}, 151 \mathrm{MHz}\right) \delta: 168.82,152.33,150.86$, $134.47,132.79,131.41,129.60,123.06,122.14,120.49$, $43.41,36.40$; IR $v: 3675,2920,744,1741,1603,1488$, 1315, 1062, 846, $719 \mathrm{~cm}^{-1}$; HRMS calcd for $\mathrm{C}_{16} \mathrm{H}_{15} \mathrm{BrN}_{6} \mathrm{SNa}[\mathrm{M}+\mathrm{Na}]^{+}$425.0160, found 425.0158.

2-(4-氟苯胺基)-5-[4-(3,3-二甲基三氮烯-1-基)苯 基]-1,3,4-噻二唑 $(80)$ : 淡黄色固体, 产率 83.8\%. m.p. $223 \sim 225{ }^{\circ} \mathrm{C} ;{ }^{1} \mathrm{H}$ NMR (DMSO- $\left.d_{6}, 600 \mathrm{MHz}\right) \delta$ : 14.09 (s, $1 \mathrm{H}), 7.44(\mathrm{dd}, J=8.7,4.9 \mathrm{~Hz}, 2 \mathrm{H}), 7.35$ (t, $J=8.8 \mathrm{~Hz}$, 2H), $7.24 \sim 7.28(\mathrm{~m}, 4 \mathrm{H}), 3.48(\mathrm{~s}, 3 \mathrm{H}), 3.13(\mathrm{~s}, 3 \mathrm{H}) ;{ }^{13} \mathrm{C}$ NMR (DMSO- $\left.d_{6}, 151 \mathrm{MHz}\right) \delta$ : 169.03, 163.31, 161.68, $152.29,151.01,131.56,129.53,122.25,120.45,116.73$, 43.40, 36.38; IR v: 3671, 2921, 2730, 1602, 1512, 1309, 1224, 1005, 839, $605 \mathrm{~cm}^{-1}$; HRMS calcd for $\mathrm{C}_{16} \mathrm{H}_{15} \mathrm{FN}_{6} \mathrm{~S}-$ $\mathrm{Na}[\mathrm{M}+\mathrm{Na}]^{+}$365.0961, found 365.0960.

\section{3 抗肿瘤活性测试}

测试所用的肿瘤细胞: 人食管癌细胞(EC109)、人胃 癌细胞(MGC803)和人前列腺癌细胞(PC-3) 由郑州大学 基础医学院药理系提供. 所有化合物均以二甲基亚砜 (DMSO)溶解配制成初浓度为 $20 \mathrm{mmol} / \mathrm{L}$ 的储备液, 于 $4{ }^{\circ} \mathrm{C}$ 冰箱中保存. 使用前用培养基稀释成所需浓度, 使 DMSO 的终含量不超过 $0.1 \%$. 将肿瘤细胞培养于改良 的 RPMI-1640 培养基, 置于 $37{ }^{\circ} \mathrm{C}$ 、体积分数为 $5 \% \mathrm{CO}_{2}$ 培养箱中培养, 计数后, 配成细胞悬液. 将细胞悬液按 每孔 $100 \mu \mathrm{L}$ 于 96 孔板中培养 $24 \mathrm{~h}$ 后, 将不同浓度的药 物加到 $200 \mu \mathrm{L}$ 培养基中, 培养 $48 \mathrm{~h}$. 取出放在超净台中 操作, 每孔加入 $20 \mu \mathrm{L} 5 \mathrm{~g} / \mathrm{L}$ 的噻唑蓝(MTT)溶液, 继续 置于培养箱中培养 $4 \mathrm{~h}$. 取出至实验台, 移除 96 孔板中 培养基, 每孔加入 $150 \mu \mathrm{L}$ 二甲基亚砜(DMSO)溶解蓝紫 色的甲瓒颗粒. 摇床混匀 $10 \mathrm{~min}$ 后用酶标仪在 $490 \mathrm{~nm}$ 处测吸光度值 $A$, 对所得数值处理, 分别计算出达卡巴 嗪和各化合物的平均值、标准差和存活率. 通过存活率 柱状图观察不同浓度药物作用的梯度情况, 用 SPSS 软 件计算每个化合物的 $\mathrm{IC}_{50}$.

\section{辅助材料(Supporting Information) 化合物 $7 \mathrm{~m}, 8 \mathrm{a} \sim$} 80 的 ${ }^{1} \mathrm{H} N \mathrm{NM} 、{ }^{13} \mathrm{C} \mathrm{NMR}$ 和 HRMS 谱图. 这些材料可以 免费从本刊网站(http://sioc-journal.cn/)上下载.

\section{References}

[1] Nan, G. M.; Zhou, J. Chin. J. Org. Chem. 2012, 32, 1695 (in Chinese). (南光明, 周均, 有机化学, 2012, 32, 1695.)

[2] Qiu, R. B.; Zhang, R. P.; He, L. F. Phys. Test. Chem. Anal., Part B 2015, 51,541 (in Chinese).

(邱如斌, 章汝平, 何立芳, 理化检验 (化学分册), 2015, 51, 541.)

[3] Li, H. F.; Lin, Q.; Kong, Y.; Li, L.; Dai, J.; Yu, W. W.; Guo, J. Tumor 2010, 30, 661 (in Chinese).

(李海夫, 林清, 孔燕, 李丽, 代杰, 于玮玮, 郭军, 肿瘤, 2010, 30, 661.)

[4] Lei, Q.; Qin, S. S.; Feng, C. N.; Li, P. P.; Zhang, X.; Long, Y. Chin. J. Org. Chem. 2016, 36, 406 (in Chinese).

(雷强, 秦上尚, 冯翠宁, 李佩佩, 张茜, 龙跃, 有机化学, 2016, 36, 406.)

[5] Lei, Q.; Zhang, S. Y.; Liu, M. L.; Li, J.; Zhang, X.; Long, Y. Mol. Diversity 2017, 21, 957.

[6] Carvalho, E.; Francisco, A. P.; Iley, J.; Rosa, E. Bioorg. Med. Chem. 2000, $8,1719$.

[7] Liu, T. T.; Wan, Y. C.; Fang, H. Chin. J. Org. Chem. 2016, 36, 417 (in Chinese) (刘婷婷, 万义超, 方浩, 有机化学, 2016, 36, 417.)

[8] Megally Abdo, N. Y.; Kamel, M. M. Chem. Pharm. Bull. (Tokyo) 2015, 63, 369.

[9] Gomha, S. M.; Kheder, N. A.; Abdelaziz, M. R.; Mabkhot, Y. N.; Alhajoj, A. M. Chem. Cent. J. 2017, 11, 25.

[10] Flefel, E. M.; El-Sayed, W. A.; Mohamed, A. M.; El-Sofany, W. I.; Awad, H. M. Molecules 2017, 22, 170.

[11] Li, Y. J.; Yu, Y.; Jin, K.; Gao, L. X.; Luo, T. C.; Sheng, L.; Shao, X.; Li, J. Chin. J. Org. Chem. 2015, 35, 129 (in Chinese). (李英俊, 于洋, 靳焜, 高立信, 罗潼川, 盛丽, 郡昕, 李佳, 有 机化学, 2015, 35, 129.)

[12] Li, S.; Jing, F.; Fu, X. Y.; Zhao, J. J.; Wang, X. F.; Li, B. L.; Liu, Y. M.; Chen, B. Q. Chin. J. Org. Chem. 2015, 35, 2624 (in Chinese). (李莎, 荆芬, 付小云, 赵继军, 王雪峰, 李宝林, 刘玉明, 陈宝 泉, 有机化学, 2015, 35, 2624.)

[13] Juszczak, M.; Matysiak, J.; Szeliga, M.; Pozarowski, P.; Niewiadomy, A.; Albrecht, J.; Rzeski, W. Bioorg. Med. Chem. Lett. 2012, $22,5466$.

[14] Aryanasab, F.; Halimehjani, A. Z.; Saidi, M. R. Tetrahedron Lett. 2010, 51,790 .

[15] Wei, G. P.; Zhang, X.; Lei, Q.; Xu, M.; Zhang, M. Q.; Long, Y. Chin. J. Org. Chem. 2018, 38, 2137 (in Chinese).

(魏光璞, 张茜, 雷强, 徐沝, 张明千, 龙跃, 有机化学, 2018, 38, 2137.)

[16] Yang, H. J.; Li, Y.; Jiang, M.; Wang, J. M.; Fu, H. Chem. Eur. J. 2011, 17, 5652.

[17] Farquhar, D.; Benvenuto, J. J. Med. Chem. 1984, 27, 1723.

[18] Giraldi, T.; Nisi, C. J. Med. Chem. 1977, 20, 850.

[19] Feng, Y.; Zou, M. M.; Song, R. J.; Shao, X. S.; Li, Z.; Qian, X. H. J. Org. Chem. 2016, 81, 10321.

[20] Yao, H. Z.; Lin, F. Y.; Chen, J. L.; Li, Y.; Cui, J. H.; Qiao, C. H. Bioorg. Med. Chem. Lett. 2016, 26, 1386.

[21] Rohde, J. U.; Kelley, M. R.; Lee, W. T. Inorg. Chem. 2008, 47, 11461.

[22] Kumavat, P. P.; Jangale, A. D.; Patil, D. R.; Dalal, K. S.; Meshram, J. S.; Dalal, D. S. Environ. Chem. Lett. 2013, 11, 177. 